\title{
Long non-coding RNA PTTG3P functions as an oncogene by sponging miR-383 and up-regulating CCND1 and PARP2 in hepatocellular carcinoma
}

\author{
Qiang Zhou', Wei Zhang ${ }^{2}$, Zhongfeng Wang ${ }^{1}$ and Songyang Liü ${ }^{2 *}$
}

\begin{abstract}
Background: Emerging evidence indicates that Long non-coding RNAs (LnCRNAs) and microRNAs (miRNAs) play crucial roles in tumor progression, including hepatocellular carcinoma (HCC). However, whether there is a crosstalk between LnCRNA pituitary tumor-transforming 3 (PTTG3P) and miR-383 in HCC remains unknown. This study is designed to explore the underlying mechanism by which LnCRNA PTTG3P sponges miR-383 during HCC progression.

Methods: qPCR and Western blot were used to analyze LncRNA PTTG3P, miR-383 and other target genes' expression. CCK-8 assay was performed to examine cell proliferation. Annexin V-PE/PI and PI staining were used to analyze cell apoptosis and cell cycle distribution by flow cytometry, respectively. Transwell migration and invasion assays were used to examine cell migration and invasion abilities. An in vivo xenograft study was performed to detect tumor growth. Luciferase reporter assay and RNA pull-down assay were carried out to detect the interaction between miR-383 and LncRNA PTTG3P. RIP was carried out to detect whether PTTG3P and miR-383 were enriched in Ago2immunoprecipitated complex.
\end{abstract}

Results: In this study, we found that PTTG3P was up-regulated in HCC tissues and cells. Functional experiments demonstrated that knockdown of PTTG3P inhibited cell proliferation, migration and invasion, and promoted cell apoptosis, acting as an oncogene. Mechanistically, PTTG3P upregulated the expression of miR-383 targets Cyclin D1 (CCND1) and poly ADP-ribose polymerase 2 (PARP2) by sponging miR-383, acting as a competing endogenous RNA (ceRNA). The PTTG3P-miR-383-CCND1/PARP2 axis modulated HCC phenotypes. Moreover, PTTG3P also affected the PI3K/Akt signaling pathway.

Conclusion: The data indicate a novel PTTG3P-miR-383-CCND1/PARP2 axis in HCC tumorigenesis, suggesting that PTTG3P may be used as a potential therapeutic target in HCC.

Keywords: Long non-coding RNA, PTTG3P, miR-383, CCND1, PARP2, Hepatocellular carcinoma

\section{Background}

Hepatocellular carcinoma (HCC) accounts for $90 \%$ of liver cancer which is the third cause of cancer-related death worldwide [1, 2]. Despite a variety of advanced therapeutic approaches, including liver resection, chemotherapy, and radiotherapy, or molecular targeted therapy, the prognosis of some HCC is still poor. Thus, it is urgent

\footnotetext{
* Correspondence: liu_songyang@126.com

${ }^{2}$ Department of Hepatobiliary and Pancreatic Surgery, the First Hospital of Jilin University, Changchun 130021, Jilin, China

Full list of author information is available at the end of the article
}

to understand the molecular mechanism of HCC tumorigenesis to explore novel biomarkers for HCC prognosis, which will promote the development of therapeutic strategy for HCC patients.

Pseudogene, a subclass of long non-coding RNAs (lncRNAs), are considered as genomic loci that resemble real gene, but lost some functionality because they are lack of protein-coding ability because of disabling mutation, lack of transcription, or their inability to encode RNA [3]. However, recent studies have revealed that pseudogene-derived lncRNAs play important roles in

(c) The Author(s). 2019 Open Access This article is distributed under the terms of the Creative Commons Attribution 4.0 International License (http://creativecommons.org/licenses/by/4.0/), which permits unrestricted use, distribution, and reproduction in any medium, provided you give appropriate credit to the original author(s) and the source, provide a link to the Creative Commons license, and indicate if changes were made. The Creative Commons Public Domain Dedication waiver (http://creativecommons.org/publicdomain/zero/1.0/) applies to the data made available in this article, unless otherwise stated. 
cellular process [4-6]. Accumulating evidence indicates that lncRNAs, longer than 200 nucleotides in length and no protein coding potentials, exert crucial roles in pathological process, including cancer development and progression [7, 8]. For example, LincDUSP regulates the colon cancer cell cycle progression and reduces the susceptibility to apoptosis [9], which is upregulated in colon cancer. LncRNA00152 promotes glioma cell proliferation and invasion via the regulation of miR-16, functioning as an oncogene [10]. MicroRNAs (miRNAs) are a family of small non-coding RNA molecules, 22 nucleotides in length, and act as important regulatory modulators of gene expression at the post-transcriptional level through the complete or incomplete base pairs between miRNAs and their targets' mRNA 3'UTR, resulting in the target mRNA degradation or translational repression [11-13]. MiRNAs are reported to involved in multiple cellular processes [14]. Bioinformatics algorithms including miRCODE (http://www.mircode.org/) suggest that miRNAs can interact with lncRNAs. A series of studies indicate that lncRNAs serve as competing endogenous RNAs (ceRNA) by sponging miRNAs, and modulate the targets of miRNAs $[15,16]$. For instance, miR-190 suppresses the EMT of hepatoma cells by targeting lncRNA treRNA [17]. LncRNA SNHG16 promotes the glioma cell proliferation and suppresses cell apoptosis via sponging miR-4518 and upregulating its target RPMI5 [18]. MEG3 inhibits human pancreatic endocrine tumor cell growth and metastasis through acting as a ceRNA of miR-183 [19]. The pseudogene-derived lncRNA PTTG3P has been reported to act as an oncogene in gastric cancer [20] and HCC [21], but the molecular mechanism how PTTG3P interacts with miRNAs in HCC remains poor.

In this study, we found that PTTG3P was upregulated in HCC. Knockdown of PTTG3P suppressed cell growth, migration and invasion, and promoted cell apoptosis by sponging miR-383 and regulating miR-383 targets, CCND1 and PARP2, as well as the PI3K/Akt signaling pathway.

\section{Methods}

\section{Tissue samples}

Fifty paired HCC and adjacent non-tumor tissue samples (within $2 \mathrm{~cm}$ of tumor) were acquired from the First Hospital of Jilin University during HCC surgery between January 2015 and July 2018. All tumor tissues were immunohistochemically validated and acquired patients' consent for the tissues used for this study. The tissues were frozen in liquid nitrogen after resection.

\section{Cell culture}

Six human HCC cell lines, HepG2, Hep3B, Huh-7, HLF, SK-HeP-1, SNU-449, and normal liver cells LO2 were obtained from the Cell Bank of Chinese Academy of
Sciences. HepG2, Hep3B, Huh-7, HLF and SK-HeP-1 cells were cultured in Dulbecco's Modified Eagle Medium (DMEM, HyClone, USA, AC10253739), while SNU-449 and LO2 cells were cultured in RPMI 1640 medium (HyClone, USA, AD12582265). All cells were supplemented with $10 \%$ fetal bovine serum (FBS, Coning, USA, 35081003), $100 \mathrm{U} / \mathrm{ml}$ penicillin and $100 \mathrm{mg} / \mathrm{ml}$ streptomycin. The cells were maintained in a humidified incubator at $37^{\circ} \mathrm{C}$ with $5 \% \mathrm{CO}_{2}$.

\section{Cell transfection}

siRNAs, miR-383 inhibitor, miR-383 mimic, or corresponding controls were acquired from GENECHEM (Shanghai, China), and were transfected into the cells by Lipofectamine $^{\mathrm{Tm}} 3000$ according to the manufacturer's instructions.

Lentivirus overexpressing shRNA against PTTG3P was obtained from GENECHEM (Shanghai, China) and infected HepG2 cells, followed by selection using puromycin.

\section{RNA isolation and qPCR}

Total RNAs were isolated using TriZol reagent (Qiagen) from tissues or transfected cells according to the manufacturer's protocols. $500 \mathrm{ng}$ of RNA was used for cDNA synthesis using the Prime Script RT regent Kit. The cDNA synthesis of PTTG3P used the random primer and Oligo $d(T)$. The cDNA synthesis of miR-383 used specific miR-383 reverse transcription primer. The fast Start Universal Green Masta (Roche) was used for qPCR on the ABI 7900 detection system. The primers were listed in Table 1. U6 snRNA was used as an internal control to normalize miR-383 expression. GAPDH was used to normalize target genes' expression. The relative gene expression was normalized to GADPH or U6 expression and calculated according to the $2^{-\Delta \Delta C q}$ method described by Livak and Schmittgen [22].

Table 1 Primers used for RT and $\mathrm{qPCR}$

\begin{tabular}{ll}
\hline miR-383 RT primer & 5'GTCGTATCCAGTGCGTGTCGTGGAGTCGGCAA \\
\hline miR-383 & Forward: 5'GGGAGATCAGAAGGTGATTGTGGCT3' \\
& Reverse: 5'CAGTGCGTGTCGTGGAGT3' \\
U6 snRNA & Forward: 5'CTCGCTTCGGCAGCACA3' \\
& Reverse: 5'AACGCTTCACGAATTGCGT3' \\
GAPDH & Forward: 5'GGAGCGAGATCCCTCCAAAAT3' \\
& Reverse: 5'GGCTGTTGTCATACTTCTCATGG3' \\
PTTG3P & Forward: 5'AAACGAAGAACCAGGCATCCTT3' \\
& Reverse: 5'GGGAGCATCGAATGTTTTGCC3' \\
CCND1 & Forward: 5'GCTGCGAAGTGGAAACCATC3' \\
& Reverse: 5'CCTCCTTCTGCACACATTTGAA3' \\
PARP2 & Forward: 5'GCCTTGCTGTTAAAGGGCAAA3' \\
& Reverse: 5'TCCTTCACACTCCACATGAGCC3' \\
\hline
\end{tabular}




\section{Western blot assay}

Cells were harvested and lysed using RIPA lysis buffer on ice for $30 \mathrm{~min}$. The lysates were then immunoblotted. The primary antibodies against CCND1 (Rabbit polyclonal to CCND1, 1:1000 dilution, Abcam), and PARP2 (Rabbit polyclonal to PARP2, 1:1000, Abcam) were used. The horseradishperoxidase (HRP)-conjugated anti-rabbit IgG antibody was used as the secondary antibody. Finally, the blots were visualized by an enhanced chemiluminescence system.

\section{CCK-8 assay}

Cell viability was determined using the Cell Counting Kit-8 (CCK-8) according to the manufacturer's instructions. Briefly, the transfected cells were seeded into the 96-well plate with a density of 3000 cells/well. CCK-8 reagent was added into the medium at $48 \mathrm{~h}$ after transfection, followed by incubation for $1 \mathrm{~h}$ at $37^{\circ} \mathrm{C}$. Finally, the absorbance was measured at $450 \mathrm{~nm}$ by a spectrometer $\left(\mathrm{OD}_{450 \mathrm{~nm}}\right)$.

\section{Cell apoptosis assay}

Cell apoptosis was analyzed using the Annexin V-PE/PI kit by flow cytometry according to the manufacturer's protocols. Annexin V-PE (+)/PI (-) represented apoptotic cells, while Annexin V-PE (+)/PI (+) represented the advance apoptotic cells or dead cells.

\section{Cell cycle analysis}

Cell cycle was measured with propidium iodide (PI) by flow cytometry. Briefly, at $48 \mathrm{~h}$ after transfection, the cells were harvested and stained with PI using the CycleTest Plus DNA Reagent kit (BD) following the manufacturer's guide. Finally, the percentage of cells in G0/G1, S and G2/M stages was counted.

\section{Transwell migration and invasion assays}

Cell migration and invasion abilities were analyzed using Transwell Chambers (Corning, $8 \mu \mathrm{m}$ pore) according to the manufacturer's protocols. Briefly, the transfected cells (25000 cells) were suspended in $200 \mu \mathrm{l}$ of serumfree medium and plated into the upper compartment. For the invasion assay, the upper compartment was precoated with Matrigel (Sigma). The lower chamber contained 10\% FBS-containing medium. After incubation for approximately $20 \mathrm{~h}$, the migrated or invaded cells were fixed and stained by crystal violet. The cells not migrated or invaded into the membrane were scraped using cotton tips.

\section{Luciferase reporter assay}

PTTG3P fragment containing miR-383 binding sites was PCR-amplified and cloned downstream of a luciferase reporter gene in the pmirGLO vector, named pmirGLO-
PTTG3P. The pmirGLO-mut PTTG3P (mutations within the binding site) was generated using the Quickchange $^{\mathrm{XL}}$ Site-directed Mutagenesis kit (Stratagene) according to the manufacturer's protocols. The cells were co-transfected with miR-383 and wild-type PTTG3P or mutant PTTG3P vector, together with controls. At $48 \mathrm{~h}$ after transfection, the cells were harvested and subjected to Luciferase assay using the Dual-luciferase reporter system (Promega) following the manufacturer's instructions. The luciferase assays for miR-383 and its targets CCND1 and PARP2 were similar to that of PTTG3P luciferase reporter system.

\section{In vivo animal study}

The animal research was approved by the ethics committee of the First Hospital of Jilin University and the Institutional Animal Care and Use Committee. 4-6 weeks old female nude mice were used for this study. Briefly, $10^{6}$ cells were suspended in mixture of PBS and Matrigel (1:1) and subcutaneously injected into the flank of the nude mice. The tumor size was measured using a Vernier caliper by the formula $V=1 / 2\left(L^{*} W^{2}\right)$, where $L$ represented the length (longest dimension), and W represented the width (shortest dimension). After about six weeks, all mice in experiments were euthanized by injection of sodium pentobarbital $(100 \mathrm{mg} / \mathrm{kg})$ followed by cervical dislocation. Finally, the xenograft tissues were used for qPCR to analyze PTTG3P expression.

\section{RNA pull-down assay}

RNA pull-down assay was performed using the Pierce ${ }^{\mathrm{Ts}}$ Magnetic RNA-Protein Pull-Down Kit according to the manufacturer's instructions. Briefly, HepG2 or Huh-7 cells were transfected with 3'end biotinylated miR-383 or mutant miR-383 or controls treated using Pierce ${ }^{\mathrm{TM}}$ RNA 3' End Desthiobiotinylation Kit, followed by incubation with streptavidin-coated magnetic heads after transfection for $24 \mathrm{~h}$. The level of PTTG3P in the bound fraction was determined by qPCR.

\section{RNA immunoprecipitation (RIP) assay}

RIP assay was performed using a RIP kit (Millipore) according to the manufacturer's protocols. Briefly, RNAs were isolated and subjected to qPCR. The antibody against Ago2 and negative control IgG were obtained from Abcam (USA).

\section{Statistical analysis}

The data were shown as mean \pm standard deviation (SD) from three independent experiments. The differences between groups were analyzed using the Student's t-test (two groups) or One-way ANOVA (multiple groups) by GraphPad Prism 5.0 software. The value of $P<0.05$ was considered statistically significant. 


\section{Results}

PTTG3P is highly expressed in HCC tissues and cell lines Firstly, we analyzed the profiles of HCC patients from the Gene Expression Omnibus (GEO) (GSE 76427 and GSE 84402 dataset), and found that PTTG3P was upregulated in HCC tissues compared to adjacent nontumor tissues. Next, we aimed to determine whether PTTG3P was overexpressed in HCC. A total of 50 paired HCC tissues were evaluated for PTTG3P expression using qPCR. As results shown in Fig. 1a, PTTG3P was indeed up-regulated in HCC tissues, compared to adjacent non-tumor samples. Moreover, we examined PTTG3P expression in HCC cell lines, Huh-7, HepG2, Hep3B, SNU-449, HLF, SK-Hep-1. PTTG3P was highly expressed in these cell lines, compared to that in the normal liver cells LO2 (Fig. 1b), in which HepG2 and Huh-7 had the lowest and highest levels. Thus, HepG2 and Huh-7 were selected for the following study.

To further investigate the relationship between PTTG3P and clinicopathological features of HCC patients, patients were divided into two groups: high and low PTTG3P levels. As data shown in Table 2, high PTTG3P level was related to tumor size, high stage and metastasis. However, PTTG3P level was not related to other factors, such as age and genders.

\section{Knockdown of PTTG3P inhibits cell proliferation and promotes cell apoptosis}

Next, we aimed to explore the biological functions of PTTG3P in cell proliferation. HepG2 and Huh-7 cells were transfected with PTTG3P siRNA, and the efficiency of siRNA was confirmed by qPCR (Fig. 2a). CCK-8 and colony formation assays were preformed to detect cell proliferation. As results shown in Fig. 2b, knockdown of
PTTG3P inhibited cell viability, compared to control group. Similar results were obtained in colony formation assay. PTTG3P knockdown suppressed the colony-forming ability (Fig. 2c). To further investigate whether the effect of PTTG3P on cell proliferation was related to cell cycle progression, we performed flow cytometry to analyze cell cycle. As shown in Fig. 2d, HepG2 and Huh-7 cells transfected with PTTG3P siRNA had a higher percentage of cells in G0/G1 stage, and a lower percentage of cells in $\mathrm{S}$ stage, suggesting that PTTG3P knockdown inhibited cell cycle G1/S transition. Finally, cell apoptosis assay showed that cells with PTTG3P siRNA had a high percentage of apoptosis, compared to control group (Fig. 2e, Additional file 2: Figure S2). Overall, these data indicate that PTTG3P knockdown inhibits cell cycle progression, cell proliferation and promotes cell apoptosis.

\section{Knockdown of PTTG3P inhibits cell migration and invasion}

Considering that metastasis is an important factor involved in tumor progression, we aimed to investigate the effect of PTTG3P on cell migration and invasion. As results shown in Fig. 3a, we found that cells transfected with PTTG3P siRNA had a lower migratory number, compared to control group. Similar results were obtained in the invasion assay which showed that PTTG3P siRNA suppressed the number of invasive cells (Fig. 3b). Taken together, the data indicate that knockdown of PTTG3P exerts tumor suppressive roles in inhibiting cell migration and invasion.

Knockdown of PTTG3P inhibits HCC tumor growth in vivo We finally examined the effect of PTTG3P on tumorigenesis in a xenograft study. HepG2 cells stably

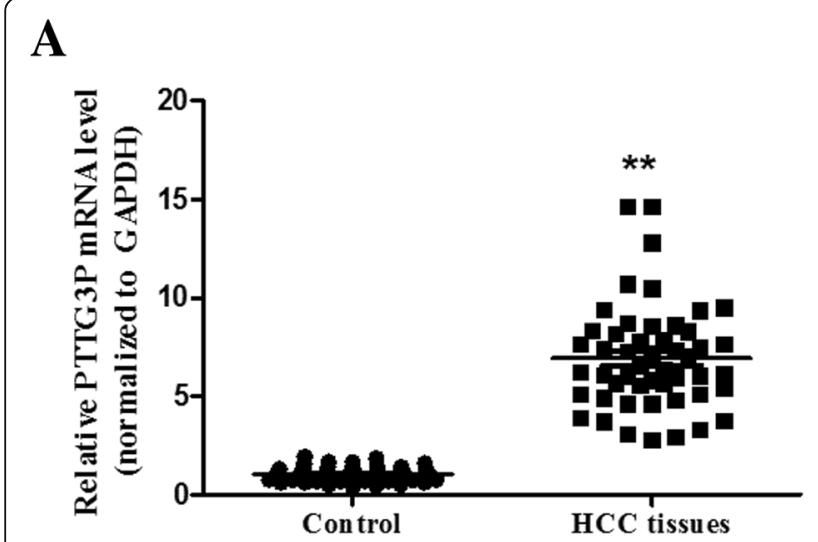

B

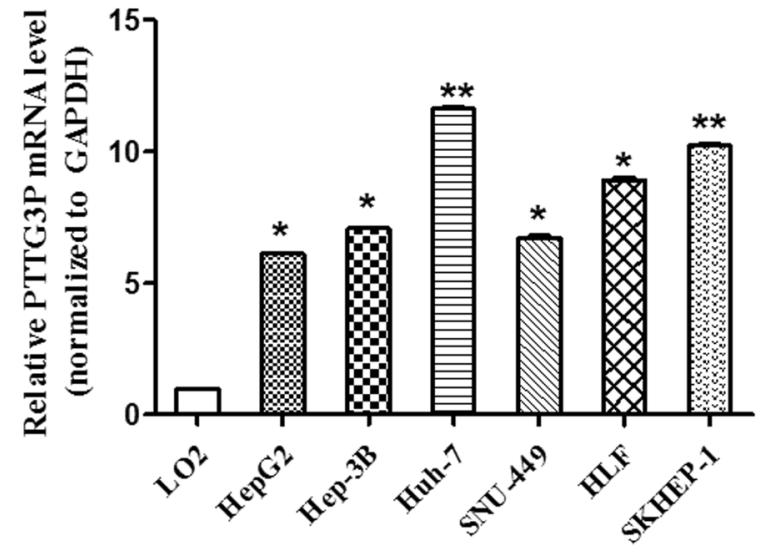

Fig. 1 PTTG3P is highly expressed in HCC tissues and cell lines. a qPCR analysis of PTTG3P expression in 50 paired HCC tissues and adjacent nontumor tissues. $\mathbf{b}$ qPCR analysis of PTTG3P expression in the normal liver cells (LO2) and HCC cells. The data were shown as mean \pm SD and the experiment was performed in triplicate. ${ }^{*} P<0.05 .{ }^{* *} P<0.01$ 
Table 2 Relationship between the clinicopathological features of HCC and PTTG3P levels

\begin{tabular}{llll}
\hline Characteristics & High & Low & $P$ \\
\hline Gender & 14 & 13 & $>0.05$ \\
Male & 12 & 11 & \\
Female & & & \\
Age & 11 & 15 & $>0.05$ \\
$>55$ & 12 & 12 & \\
$<=55$ & & & $<0.001$ \\
Tumor size (cm) & 5 & 13 & \\
$<=5$ & 25 & 7 & $<0.001$ \\
$>5$ & & 17 & $<0.001$ \\
Stage & 8 & 4 & \\
I-II & 21 & 15 & \\
III-IV & & 4 & \\
Metastasis & 2 & & \\
Negative & 29 & & \\
Positive & & & \\
\hline
\end{tabular}

overexpressing PTTG3P shRNA were subcutaneously injected into the flank of the nude mice. Tumor sizes were measured every week. As shown Fig. $3 c$ and d, we found that the xenograft formed by PTTG3P shRNAtreated cells had a smaller tumor size compared to control group. We also analyzed the expression of PTTG3P in the xenografts to confirm the delivery of PTTG3P shRNA into the mice. The results from the Fig. 3e showed that PTTG3P was downregulated in the xenografts formed by the PTTG3P shRNA transfected cells.

\section{PTTG3P could directly target miR-383 in HCC cells}

Accumulating evidence demonstrates that LncRNAs can function as competing endogenous RNAs (ceRNAs) or sponge in modulating the biological functions of miRNAs [23]. Using the bioinformatics algorithms miRCODE (http://mircode.org/), we found that there existed potential interactions between PTTG3P and miR-383 (Fig. 4a). Luciferase reporter assay was performed to determine the interaction between PTTG3P and miR-383. As shown in Fig. 4b, the results indicated that miR-383 reduced the luciferase intensity controlled by PTTG3P, while miR-383 did not affect the luciferase intensity of
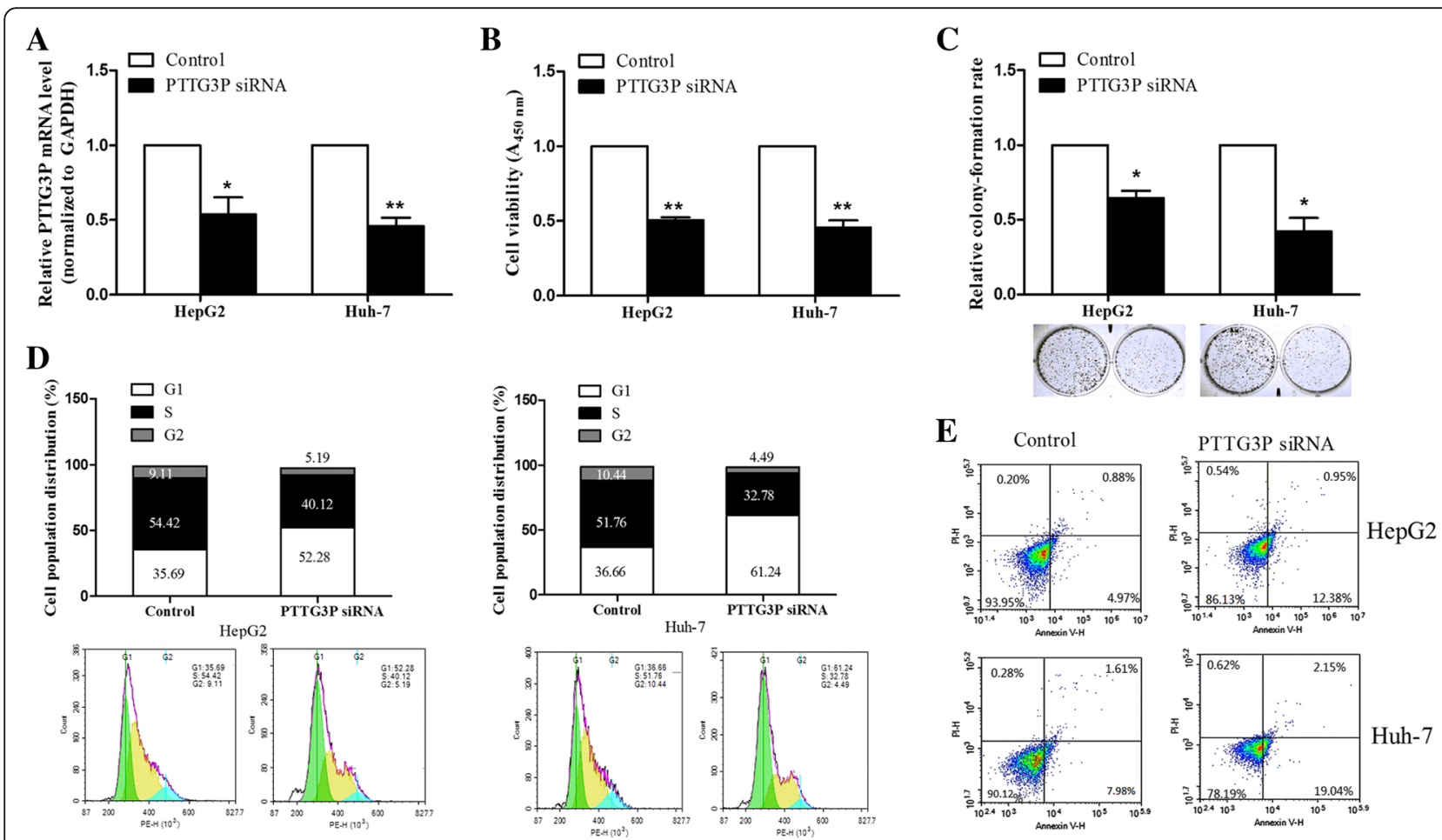

Fig. 2 Knockdown of PTTG3P suppresses cell proliferation and promotes cell apoptosis. a qPCR analysis of PTTG3P expression in PTTG3P siRNAtransfected HepG2 and Huh-7 cells. b CCK-8 assay was used to detect the viability of PTTG3P siRNA in HepG2 and Huh-7 cells. c Colony formation assay was performed to detect the colony-forming ability of PTTG3P siRNA in HepG2 and Huh-7 cells. Colonies were counted and captured. d Flow cytometry was performed to investigate the cell cycle distribution of HepG2 and Huh-7 cells. The color code represented the G1 stage, S stage and G2 stage, respectively (Left to right). e Flow cytometry was performed to detect the apoptosis of HepG2 and Huh-7 cells. LR, early apoptotic cells; UR, late apoptotic cells or dead cells. The results were shown as mean \pm SD from three independent experiments and one of the representative results was shown. Each experiment was performed in triplicate. ${ }^{*} P<0.05 .{ }^{* *} P<0.01$ 
A
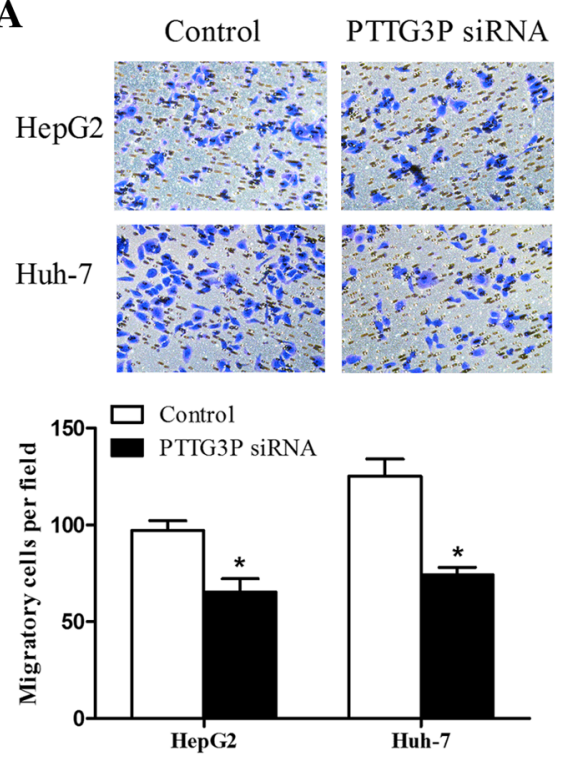

C

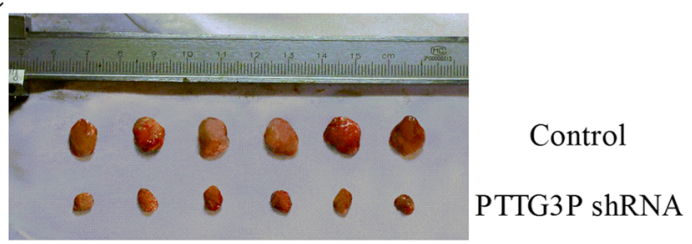

D

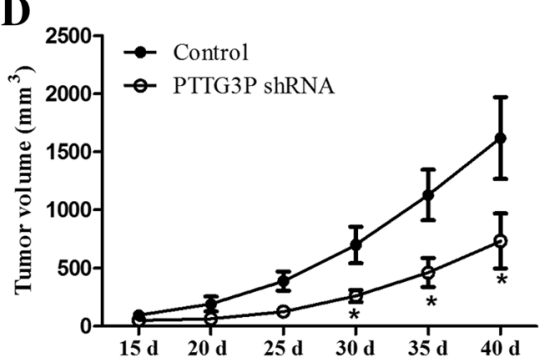

B
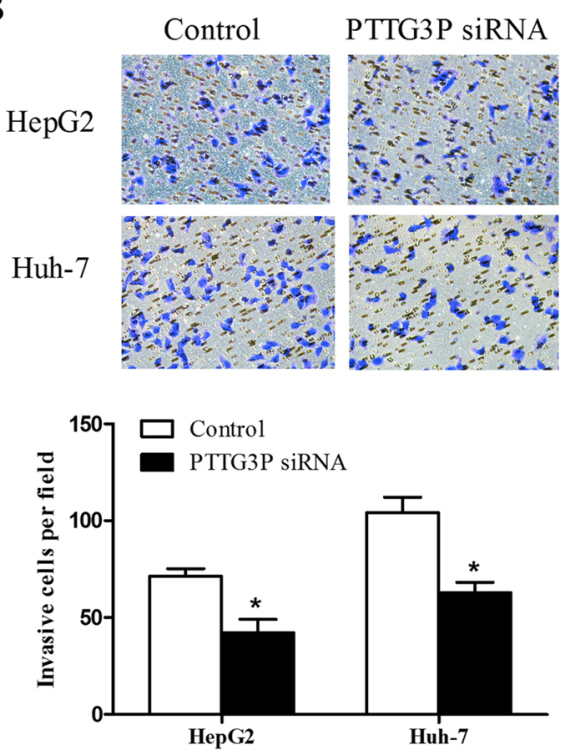

$\mathbf{E}$

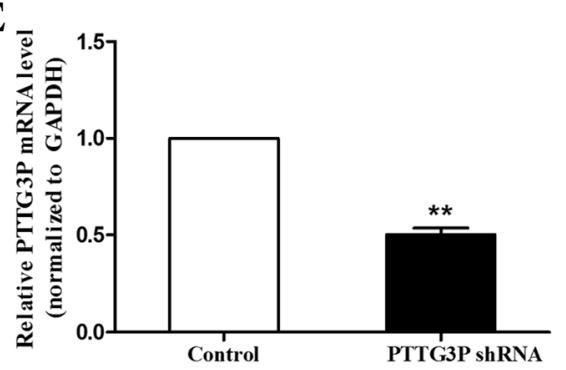

Fig. 3 Knockdown of PTTG3P suppresses the cell migration, invasion and tumor growth in vivo. a, b Transwell assays were preformed to detect the migratory (a) and invasive (b) abilities of PTTG3P siRNA-transfected or siRNA-control-transfected HepG2 and Huh-7 cells. Migratory and invasive cells were counted and captured. The results were shown as mean \pm SD from three independent experiments and one of the representative results was shown. Each experiment was performed in triplicate. c HCC cells infected with shRNA-PTTG3P or control were injected into nude mice, respectively. Tumor xenografts in sh-PTTG3P group were smaller than the control group. $\mathbf{d}$ Tumor volumes were measured after injection every week. e qPCR analysis of PTTG3P expression in xenograft tumors. ${ }^{*} P<0.05 .{ }^{* *} P<0.01$

mutant PTTG3P, compared to the control group. Similar results were shown in Huh-7 cells and HepG2 cell. Moreover, we found that PTTG3P overexpression suppressed miR-383 expression level, while PTTG3P knockdown increased miR-383 level (Fig. 4c). Finally, miR-383 levels were examined in 50 paired HCC tissues and adjacent non-tumor tissues. As shown in Fig. 4d, miR-383 was downregulated in HCC tissues. Importantly, there existed a negative correlation between miR-383 and PTTG3P levels. Finally, we performed RNA pull-down assay to validate the interaction between PTTG3P and miR-383. As shown in Fig. 4e, RNA pull-down assay demonstrated that PTTG3P was more enriched in miR383 compared to that in mutant miR-383 with broken PTTG3P binding site. In addition, RIP assay indicated that both PTTG3P and miR-383 were enriched in Ago2-containg miRNA ribonucleoprotein complexes compared to IgG immunoprecipitates (Fig. 4f). These data suggest that miR-383 can directly target PTTG3P in HCC.

\section{PTTG3P/miR-383/CCND1 or PARP2 axis modulates HCC} cell growth, migration and invasion

Considering that miR-383 was a target of PTTG3P in HCC cells, we tried to elucidate whether the potential 
A

Chr8: 67680226-67680231 (-)

PTTG3P 5' AATCCAGAATGGCTACTCTGATCT3'

B
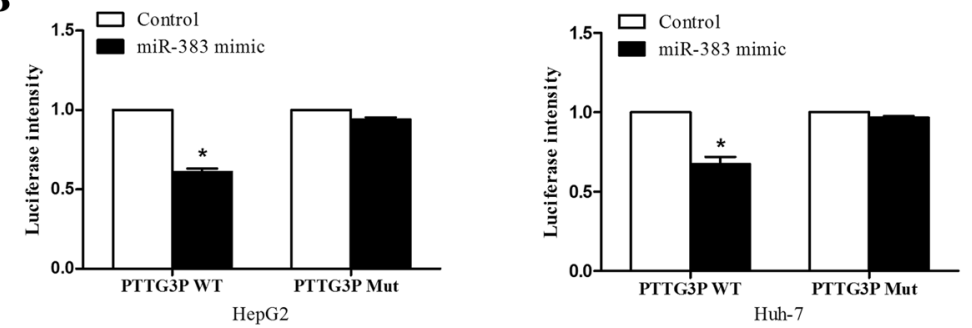

C
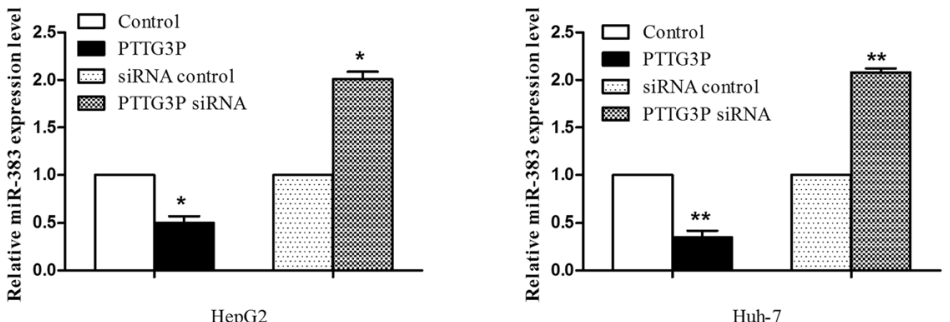

D
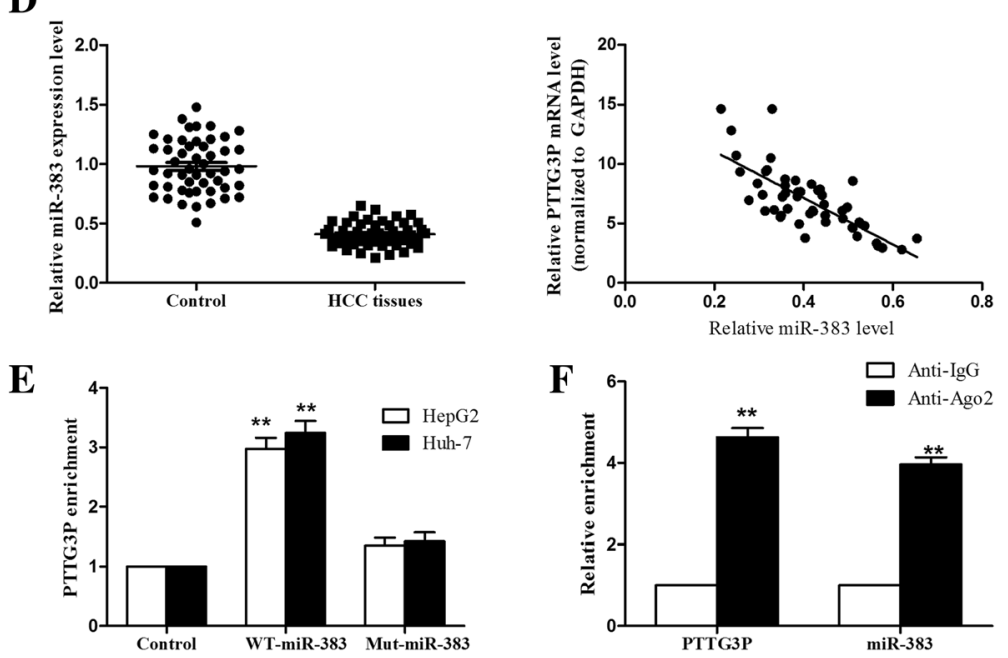

Fig. 4 PTTG3P directly interacts with miR-383 in HCC cells. a The alignment between PTTG3P and miR-383. b Luciferase reporter assay was performed to detect the effect of miR-383 on luciferase intensity controlled by PTTG3P or mutant PTTG3P. c qPCR was used to analyze miR-383 expression in HepG2 and Huh-7 cells transfected with PTTG3P, or PTTG3P siRNA or controls. $\mathbf{d}$ miR-383 expression was analyzed by qPCR in 50 paired HCC tissues and adjacent non-tumor tissues. In addition, there was a negative correlation between miR-383 and PTTG3P expression in HCC. e RNA pull-down assay was performed to determine the interaction between PTTG3P and miR-383 in HCC cells. The biotinylated miR-383 or mutant miR-383 was transfected into HCC cells, and PTTG3P expression was analyzed by qPCR. f Anti-Ago2 RIP was used to detect the miR-383 and PTTG3P enrichment in immunoprecipitates in HCC cells. IgG was used as a negative control. The results were shown as mean \pm SD from three independent experiments and one of the representative results was shown. Each experiment was performed in triplicate. ${ }^{*} P<0.05$. ${ }^{* *} P<0.01$

ceRNA mechanism among LncRNA PTTG3p, miR-383 and miR-383's targets do exist. Firstly, we performed rescue experiments to observe the roles of PTTG3P/miR383 in regulating HCC cells growth, migration and invasion. HepG2 and Huh-7 cells were co-transfected with PTTG3P siRNA and miR-383 inhibitor or controls, and subjected to functional experiments. As results shown in Fig. 5a, we found that inhibition of miR-383 ameliorated the anti-growth of PTTG3P knockdown, compared to control group. The cell cycle analysis indicated that inhibition of miR-383 promoted G1/S transition that was suppressed by PTTG3P knockdown 
A

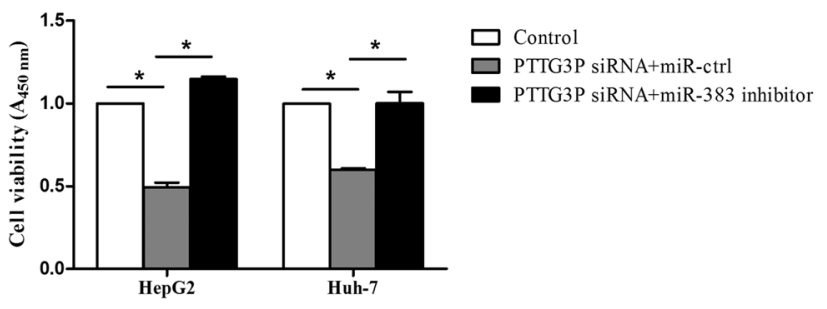

B
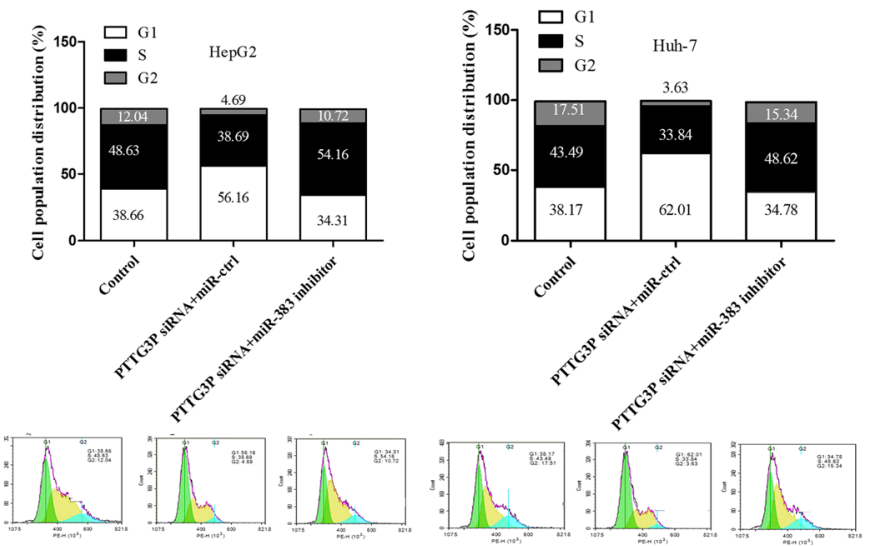

C

D
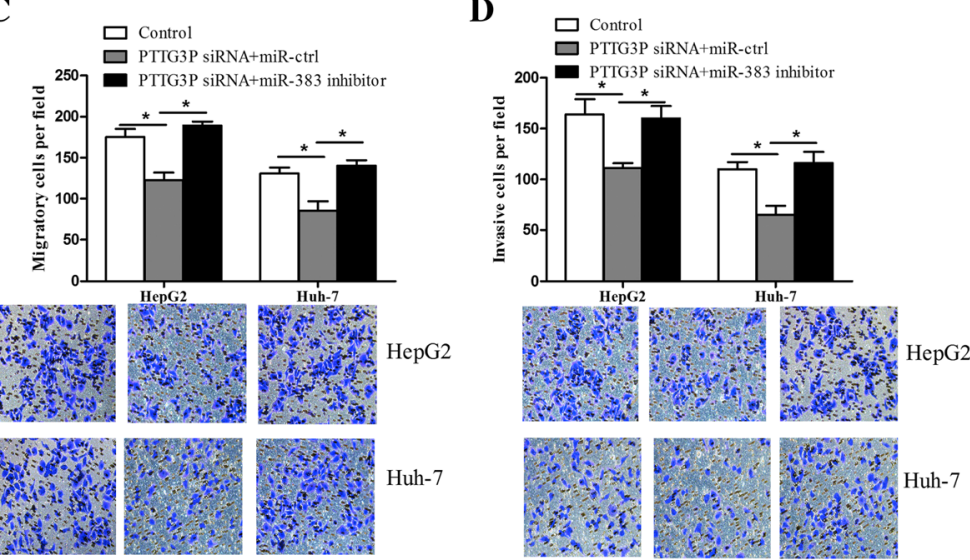

Fig. 5 PTTG3P/miR-383 axis in the phenotypes of HCC cells. HepG2 and Huh-7 cells were co-transfected with PTTG3 siRNA and miR-383 inhibitor, or corresponding controls, and then subjected to CCK-8 assay to detect the cell viability (a), or to flow cytometry to detect the cell cycle distribution (b), or to Transwell migration (c) and invasion (d) assays to detect the changes in the migratory and invasive abilities of HCC cells. The color code in 5B represented the G1 stage, S stage and G2 stage, respectively (Left to right). The results were shown as mean \pm SD from three independent experiments and one of the representative results was shown. Each experiment was performed in triplicate. ${ }^{*} P<0.05$

(Fig. 5b). Similarly, the data from Transwell migration and invasion assays showed that miR-383 inhibition enhanced the number of migratory and invasive cells which was reduced by PTTG3P knockdown (Fig. 5c and d). These data illustrate that miR-383 inhibition abolishes the antigrowth and anti-metastasis activities of PTTG3P knockdown in HCC cells.

We next determined whether PTTG3P can modulate the expression of miR-383 targets in HCC cells. CCND1 and PARP2 are validated miR-383 targets as previously described [24, 25]. As shown in Fig. 6a, we found that PTTG3P overexpression increased CCND1 mRNA levels in HepG2 and Huh-7 cells, while miR-383 overexpression can restore CCND1 expression increased by PTTG3P. However, mutant PTTG3P did not affect CCND1 expression when the binding sites of miR-383 were mutated. Similar tendency was shown in PARP2 expression levels (Fig. 6b). By contrast, knockdown of PTTG3P reduced CCND1 and PARP2 expression levels, while miR-383 inhibition can abrogate the inhibitory effect of PTTG3P on CCND1 and PARP2 (Fig. 6c and d). The protein levels of CCND1 and PARP2 were in line with that of CCND1 and PARP2 mRNA (Fig. 6e-h, Additional file 1: Figure S1A-D). The luciferase assay 
A

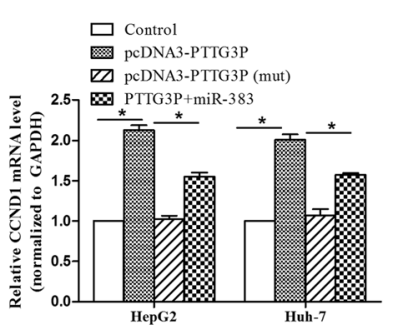

C $\square$ Control

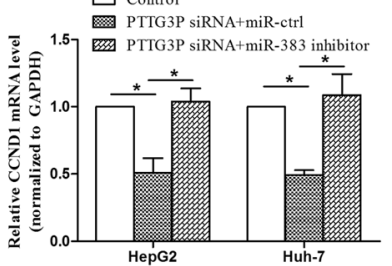

E

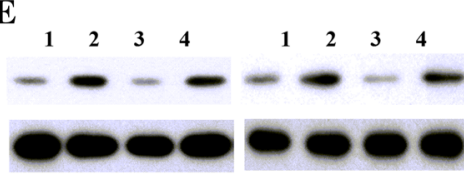

HepG2

Huh-7

1-Control

2-pcDNA3-PTTG3P

3-PTTG3P mut

4-PTTG3P+miR-383

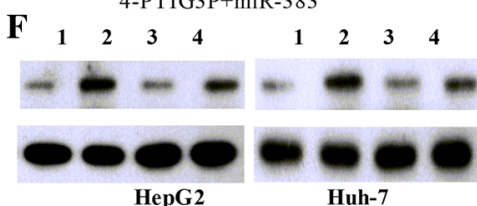

I

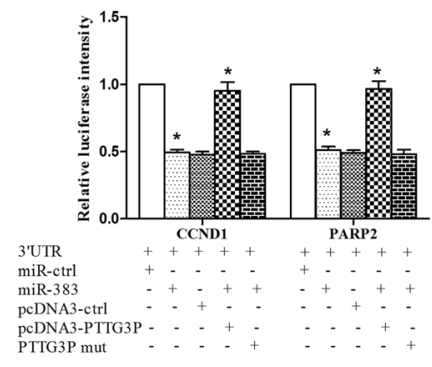

B

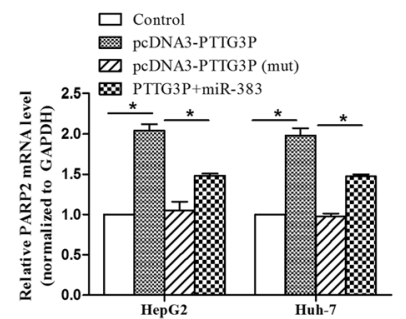

D

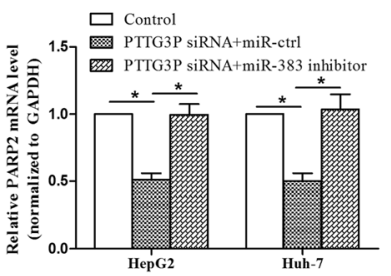

G

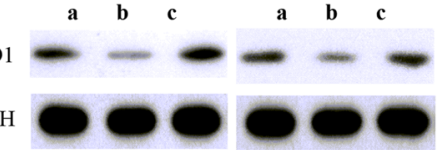

HepG2

a-Control

b-PTTG3P siRNA+miR-ctrl

c-PTTG3P siRNA

+miR-383 inhibitor
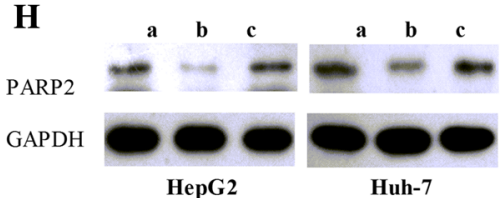

J

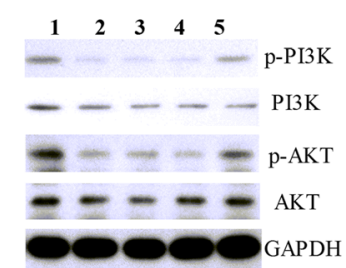

1-control 2-PTTG3P siRNA

3-CCND1 siRNA 4-PARP2 SiRNA

5-PTTG3P siRNA+miR-383

inhibitor

Fig. 6 PTTG3P affects the expression of miR-383 targets in HCC cells. a, b HepG2 and Huh-7 cells were transfected with PTTG3P, or mutant PTTG3P, or PTTG3P and miR-383, or control vector, and then QPCR was used to detect CCND1 (a) and PARP2 (b) expression. $\mathbf{c}, \mathbf{d}$ HepG2 and Huh-7 cells were transfected with PTTG3P siRNA, or PTTG3P siRNA and miR-383 inhibitor, or controls, and then qPCR was performed to detect CCND1 (c) and PARP2 (d) expression. e, f HepG2 and Huh-7 cells were transfected with PTTG3P, or mutant PTTG3P, or PTTG3P and miR-383, or control vector, and then Western blot assay was used to detect CCND1 (e) and PARP2 (f) expression. c, d HepG2 and Huh-7 cells were transfected with PTTG3P siRNA, or PTTG3P siRNA and miR-383 inhibitor, or controls, and then APCR was performed to detect CCND1 (g) and PARP2 (h) expression. i Luciferase reporter assay was performed to detect CCND1 3'UTR or PARP2 3'UTR intensity in the cells co-transfected with target 3'UTR and miR-383 and PTTG3P, or mutant PTTG3P. $\mathbf{j}$ Western blot assay was performed to analyze the phosphorylation of PI3K/Akt signaling pathway in cells transfected with siRNA against PTTG3P, or CCND1, or PARP2, or PTTG3P siRNA and miR-383 inhibitor. The results were shown as mean \pm SD from three independent experiments and one of the representative results was shown. Each experiment was performed in triplicate. ${ }^{*} P<0.05$

indicated that miR-383 reduced the luciferase intensity controlled by CCND1 3'UTR, while PTTG3P but not mutant PTTG3P abolished the inhibitory roles of miR-383 in CCND1 3'UTR intensity (Fig. 6i). In line with the effect of miR-383 on CCND1, similar results were shown in
PARP2-related assays. Finally, we found that siRNA against PTTG3P or CCND1 or PARP2 inhibited the phosphorylation of PI3K and Akt, while miR-383 inhibition was cotransfected, the inhibitory roles of PTTG3P knockdown were abolished (Fig. 6j, Additional file 1: Figure S1E). 


\section{Discussion}

Accumulating evidence indicates that aberrant LncRNA expression is related to HCC tumorigenesis, such as HOTAIR [26], MALAT1 [27] and TUG1 [28]. In this study, we found that pseudogene-derived LncRNA PTTG3P was upregulated in HCC tissues and cells, and high PTTG3P level was related to tumor size and metastasis. Knockdown of PTTG3P suppressed cell cycle progression, cell proliferation, migration and invasion, and promoted cell apoptosis, functioning as an oncogene. In line with some data obtained in our study, previous research shows that PTTG3P is highly expressed in gastric cancer tissues, and promotes cell proliferation, migration and invasion in vitro and in vivo, functioning as an independent negative prognostic biomarker [20]. Another study indicates that PTTG3P is upregulated in HCC tissues and promotes cell growth and metastasis through activating PI3K/Akt pathway [21]. However, the miRNA involved in PTTG3P-mediated HCC development is not revealed.

As gene regulators, LncRNAs modulate gene expression via a variety of mechanism, and one of the main mechanism is to harbor miRNA to serve as a sponge to up or downregulate miRNA expression. In this study, the bioinformatics analysis indicated the potential interaction between miR-383 and PTTG3P. Luciferase assay validated that PTTG3P was a direct target of miR-383, together with the RNA pull-down and RIP assays, and miR-383 was downregulated in HCC tissues. MiR-383 modulated the functions of PTTG3P in tumor malignant phenotypes. LncRNA plays crucial roles partially via acting as a ceRNA and modulating the expression of miRNA targets as previously described [29-31]. Accordingly, as a sponge of miR-383, PTTG3P upregulated the mRNA and protein expressions of miR-383 targets, CCND1 and PARP2, while mutant PTTG3P (the binding sites between miR-383 and PTTG3P were mutated) did not affect CCND1 and PARP2 expression levels, as well as the luciferase intensity. CCND1 and PARP2 are reported to be involved in tumor development and act as oncogenes. For example, CCND1 promotes the colon cancer development via PI3K/Akt pathway [32]. MiR503 suppresses cell proliferation, migration and invasion via suppressing CCND1 expression in breast cancer [33] and ESCC [34]. MiR-383 suppresses cervical cancer cell proliferation, invasion and metastasis by inhibiting PI3K/Akt pathway via the downregulating of PARP2 [25]. In line with the findings in the above stidues, our data showed that knockdown of CCND1 and PARP2 suppressed the phophorylation of PI3K and Akt, similar to that of PTTG3P knockdown, while miR-383 inhibition can restore the inhibitory effects of PTTG3P knockdown on OI3K/Akt pathway.

\section{Conclusion}

In summary, we identified a PTTG3P-miR-383-CCND1/ PARP2 axis in HCC pathogenesis. PTTG3P acted as an oncogenic lncRNA to promote HCC development through upregulating CCND1 and PARP2 as well as PI3K/Akt pathway via sponging miR-383. The data suggest that PTTG3P may serve as a potential therapeutic target in $\mathrm{HCC}$.

\section{Additional files}

Additional file 1: Figure S1. Relative protein expression of CCND1 (A, C) and PARP2 $(B, D)$ as well as phosphorylation level of PI3K and Akt (E). ${ }^{*} P<0.05$. (TIF $763 \mathrm{~kb}$ )

Additional file 2: Figure S2. The percentage of apoptotic cells in HepG2 and Huh-7 cells after treatment with PTTG3P siRNA or control. ${ }^{*} P<0.05$. (TIF $104 \mathrm{~kb}$ )

\section{Abbreviations}

3'UTR: 3'untranslated region; CCK-8: Cell Counting Kit-8; CCND1: Cyclin D1; FBS: Fetal bovine serum; HCC: Hepatocellular carcinoma; HRP: Horseradishperoxidase; LnCRNA: Long non-coding RNA; miRNA: microRNA; PARP2: Poly ADP-ribose polymerase 2; PTTG3P: Pituitary tumor-transforming 3

\section{Acknowledgements}

The authors would like to thank all the doctors of the Department of Hepatology, and the Department of Hepatobiliary and Pancreatic Surgery, the First Hospital of Jilin University, for providing all the necessary information required for this study.

\section{Authors' contributions}

QZ and WZ performed the molecular studies. QZ and ZFW performed the animal experiments. QZ, WZ and ZFW provided experimental technical support and performed the statistical analysis. SYL designed the study and helped to draft the manuscript. All authors read and approved the final manuscript.

\section{Funding}

The study was supported by the Horizontal topics of Jilin University (3R218Q783428). The funding body did not directly participate the design of the study, the collection, analysis and interpretation of the data or the writing of the manuscript.

\section{Availability of data and materials}

All data generated or analyzed during this study are included in this published article or are available from the corresponding author on reasonable request.

\section{Ethics approval and consent to participate}

This study involving human tissues received the approval of the Ethics Board of the first hospital of Jilin University (2018-2018-386). The Animal experiments were approved by the Ethics Committee of the first hospital of Jilin University. Written informed consent was obtained from each individual participant.

\section{Consent for publication}

Not applicable.

\section{Competing interests}

The authors declare that they have no competing interests.

\section{Author details}

${ }^{1}$ Department of Hepatology, the First Hospital of Jilin University, Changchun 130021, Jilin, China. ${ }^{2}$ Department of Hepatobiliary and Pancreatic Surgery, the First Hospital of Jilin University, Changchun 130021, Jilin, China. 
Received: 26 March 2019 Accepted: 12 July 2019

Published online: 24 July 2019

\section{References}

1. Yang JD, Roberts LR. Hepatocellular carcinoma: A global view. Nat Rev Gastroenterol Hepatol. 2010;7:448-58.

2. Seino S, Tsuchiya A, Watanabe $Y$, Kawata $Y$, Kojima Y, Ikarashi S, Yanai H, Nakamura K, Kumaki D, Hirano M, et al. Clinical outcome of hepatocellular carcinoma can be predicted by the expression of hepatic progenitor cell markers and serum tumour markers. Oncotarget. 2018;9:21844-60.

3. Jacq C, Miller JR, Brownlee GG. A pseudogene structure in 5S DNA of Xenopus laevis. Cell. 1977;12:109-20.

4. Ma HW, Xie M, Sun M, Chen TY, Jin RR, Ma TS, Chen QN, Zhang EB, He XZ, De W, et al. The pseudogene derived long noncoding RNA DUXAP8 promotes gastric cancer cell proliferation and migration via epigenetically silencing PLEKHO1 expression. Oncotarget. 2017;8:52211-24.

5. Wei CC, Nie FQ, Jiang LL, Chen QN, Chen ZY, Chen X, Pan X, Liu ZL, Lu BB, Wang ZX. The pseudogene DUXAP10 promotes an aggressive phenotype through binding with LSD1 and repressing LATS2 and RRAD in non small cell lung cancer. Oncotarget. 2017;8:5233-46.

6. Liu Q, Hu X, Zhang X, Dai L, Duan X, Zhou C, Ao Y. The TMSB4 pseudogene LncRNA functions as a competing endogenous RNA to promote cartilage degradation in human osteoarthritis. Mol Ther. 2016;24:1726-33.

7. Liu X, Liang Y, Song R, Yang G, Han J, Lan Y, Pan S, Zhu M, Liu Y, Wang Y, et al. Long non-coding RNA NEAT1-modulated abnormal lipolysis via ATGL drives hepatocellular carcinoma proliferation. Mol Cancer. 2018;17:90.

8. Shen F, Cai WS, Feng Z, Chen JW, Feng JH, Liu QC, Fang YP, Li KP, Xiao HQ, Cao J, et al. Long non-coding RNA SPRY4-IT1 pormotes colorectal cancer metastasis by regulate epithelial-mesenchymal transition. Oncotarget. 2017; 8:14479-86.

9. Forrest ME, Saiakhova A, Beard L, Buchner DA, Scacheri PC, LaFramboise T, Markowitz S, Khalil AM. Colon Cancer-upregulated Long non-coding RNA lincDUSP regulates cell cycle genes and potentiates resistance to apoptosis. Sci Rep. 2018;8:7324.

10. Chen X, Li D, Gao Y, Tang W, Iw L, Cao Y, Hao B. Long intergenic noncoding RNA 00152 promotes glioma cell proliferation and invasion by interacting with MiR-16. Cell Physiol Biochem. 2018;46:1055-64.

11. Yekta S, Shih $\mathbb{H}$, Bartel DP. MicroRNA-directed cleavage of HOXB8 mRNA. Science. 2004:304:594-6.

12. Brennecke J, Stark A, Russell RB, Cohen SM. Principles of microRNA-target recognition. PLoS Biol. 2005:3:e85

13. Bartel DP. MicroRNAs: genomics, biogenesis, mechanism, and function. Cell. 2004;116:281-97.

14. Ambros $V$. The functions of animal microRNAs. Nature. 2004;431:350-5.

15. Schmitt AM, Chang HY. Long noncoding RNAs in Cancer pathways. Cancer Cell. 2016:29:452-63.

16. Bach DH, Lee SK. Long noncoding RNAs in cancer cells. Cancer Lett. 2018; 419:152-66.

17. Wang X, Ren Y, Yang X, Xiong X, Han S, Ge Y, Pan W, Zhou L, Yuan Q, Yang M. miR-190a inhibits epithelial-mesenchymal transition of hepatoma cells via targeting the long non-coding RNA treRNA. FEBS Lett. 2015;589:4079-87.

18. Lu YF, Cai XL, Li ZZ, Lv J, Xiang YA, Chen JJ, Chen WJ, Sun WY, Liu XM, Chen JB. LncRNA SNHG16 functions as an oncogene by sponging MiR-4518 and up-regulating PRMT5 expression in glioma. Cell Physiol Biochem. 2018; 45:1965-75

19. Zhang YY. Feng HM. MEG3 suppresses human pancreatic neuroendocrine tumor cells growth and metastasis by Down-regulation of Mir-183. Cell Physiol Biochem. 2017:44:345-56.

20. WA-Ohoo W, Ni S, Wang Y, Xu M, Zhang Q, Yang Y, Wu Y, Xu Q, Qi P, Tan $C$, et al. PTTG3P promotes gastric tumour cell proliferation and invasion and is an indicator of poor prognosis. J Cell Mol Med. 2017;21:3360-71.

21. Huang JL, Cao SW, Ou QS, Yang B, Zheng SH, Tang J, Chen J, Hu YW, Zheng L, Wang Q. The long non-coding RNA PTTG3P promotes cell growth and metastasis via up-regulating PTTG1 and activating PI3K/AKT signaling in hepatocellular carcinoma. Mol Cancer. 2018;17:93.

22. Livak KJ, Schmittgen TD. Analysis of relative gene expression data using real-time quantitative $P C R$ and the 2(-Delta Delta $C(T))$ method. Methods. 2001;25:402-8.

23. Cesana M, Cacchiarelli D, Legnini I, Santini T, Sthandier O, Chinappi M Tramontano A, Bozzoni I. A long noncoding RNA controls muscle differentiation by functioning as a competing endogenous RNA. Cell. 2011; 147:358-69.

24. Xu Z, Zeng X, Tian D, Xu H, Cai Q, Wang J, Chen Q. MicroRNA-383 inhibits anchorage-independent growth and induces cell cycle arrest of glioma cells by targeting CCND1. Biochem Biophys Res Commun. 2014;453:833-8.

25. Teng P, Jiao Y, Hao M, Tang X. microRNA-383 suppresses the PI3K-AKTMTOR signaling pathway to inhibit development of cervical cancer via down-regulating PARP2. J Cell Biochem. 2018;119:5243-52.

26. Hu J, Wang Z, Shan Y, Pan Y, Ma J, LA-Ohoo J. Long non-coding RNA HOTAIR promotes osteoarthritis progression via miR-17-5p/FUT2/betacatenin axis. Cell Death Dis. 2018:9:711.

27. Tao F, Tian X, Ruan S, Shen M, Zhang Z. miR-211 sponges IncRNA MALAT to suppress tumor growth and progression through inhibiting PHF19 in ovarian carcinoma. FASEB J. 2018. https://doi.org/10.1096/fj.201800495RR.

28. Sun J, Hu J, Wang G, Yang Z, Zhao C, Zhang X, Wang J. LncRNA TUG1 promoted KIAA1199 expression via miR-600 to accelerate cell metastasis and epithelial-mesenchymal transition in colorectal cancer. J Exp Clin Cancer Res. 2018;37:106

29. Xia T, Chen S, Jiang Z, Shao Y, Jiang X, Li P, Xiao B, Guo J. Long noncoding RNA FER1L4 suppresses cancer cell growth by acting as a competing endogenous RNA and regulating PTEN expression. Sci Rep. 2015;5:13455.

30. Liang WC, Fu WM, Wong CW, Wang Y, Wang WM, Hu GX, Zhang L, Xiao LJ, Wan DC, Zhang JF, et al. The IncRNA H19 promotes epithelial to mesenchymal transition by functioning as miRNA sponges in colorectal cancer. Oncotarget. 2015;6:22513-25.

31. Chen L, Wang W, Cao L, Li Z, Wang X. Long non-coding RNA CCAT1 acts as a competing endogenous RNA to regulate cell growth and differentiation in acute myeloid leukemia. Mol Cells. 2016;39:330-6.

32. Chen Y, Jiang J, Zhao M, Luo X, Liang Z, Zhen Y, Fu Q, Deng X, Lin X, Li L, et al. microRNA-374a suppresses colon cancer progression by directly reducing CCND1 to inactivate the PI3K/AKT pathway. Oncotarget. 2016;7: 41306-19.

33. Long J, Ou C, Xia H, Zhu Y, Liu D. MiR-503 inhibited cell proliferation of human breast cancer cells by suppressing CCND1 expression. Tumor Biol. 2015:36:8697-702

34. Jiang $L$, Zhao Z, Zheng $L$, Xue $L$, Zhan $Q$, Song $Y$. Downregulation of miR503 promotes ESCC cell proliferation, migration, and invasion by targeting cyclin D1. Genom Proteom Bioinf. 2017;15:208-17.

\section{Publisher's Note}

Springer Nature remains neutral with regard to jurisdictional claims in published maps and institutional affiliations.

Ready to submit your research? Choose BMC and benefit from:

- fast, convenient online submission

- thorough peer review by experienced researchers in your field

- rapid publication on acceptance

- support for research data, including large and complex data types

- gold Open Access which fosters wider collaboration and increased citations

- maximum visibility for your research: over $100 \mathrm{M}$ website views per year

At $\mathrm{BMC}$, research is always in progress.

Learn more biomedcentral.com/submissions 